Trabajos y Comunicaciones, 2da. Época, No 46, e040, octubre 2017. ISSN 2346-8971

Universidad Nacional de La Plata.

Facultad de Humanidades y Ciencias de la Educación.

Departamento de Historia

\title{
Los debates conceptuales respecto de la salud sexual y reproductiva: zun problema de derechos humanos?
}

\section{Conceptual debates on sexual and reproductive health: a human rights problem?}

\author{
Natacha Mateo * \\ * Universidad Nacional de Mar del Plata - Centro de Estudios Históricos, Argentinav | \\ mateonatacha@gmail.com
}

\section{PALABRAS CLAVE}

Derechos sexuales y reproductivos

Derechos humanos

Salud reproductiva

\section{RESUMEN}

El objetivo de este trabajo es realizar un recorrido historiográfico por los debates respecto de los derechos sexuales y reproductivos desde mediados del siglo XX hasta la actualidad, en América Latina y particularmente en Argentina. Nos interesa señalar puntualmente dos momentos de inflexión: el momento en que la salud reproductiva pasó de la agenda de población a la agenda de los derechos humanos, y la propuesta conceptual de Brown (2014) respecto de los derechos sexuales y (no) reproductivos.

\section{KEYWORDS ABSTRACT}

Sexual and reproductive rights

The objective of this work is to make a historiographic tour of the debates on sexual and reproductive rights from the middle of the 20th century to the present day in Latin America and products in Argentina. We are interested in timely pointing out the moments

Human rights

Reproductive health of inflection: the moment when reproductive health passed from the population's agenda to the human rights agenda, and Brown's (2014) conceptual proposal regarding sexual and (non) reproductive rights. 
I.

Este artículo parte de comprender que todo concepto tiene alcances y limitaciones. Sin embargo, estas no le son inherentes de por sí, sino que son el resultado de los complejos debates que acuñaron dicho concepto. Si toda concepción es resultado de un proceso de disputa constante, para comprenderlo es necesario analizar la génesis de los mismos.

Por lo tanto, nos interesa pensar respecto de los avances y retrocesos que han tenido los derechos sexuales y reproductivos desde la última mitad del siglo XX. Mientras que en 1948 se firmaba la Declaración Universal de los Derechos Humanos, la discusión sobre la natalidad y la fecundidad en Argentina formaba parte de los debates poblacionales de la época sin contemplar el deseo de las mujeres. En 1968, en la Conferencia Internacional de Derechos Humanos se resolvió que la elección de cuántos hijos tener y cuándo era un derecho de las personas. Sin embargo, en 1974 en Argentina, José López Rega decretó, con fines poblacionistas, la prohibición de la comercialización de pastillas anticonceptivas y el cierre de los consultorios públicos de planificación familiar. Recién en la década de 1980 se comenzó a mencionar en el mundo el concepto de Derechos Reproductivos, aunque aún estábamos lejos de poder hablar de Derechos no Reproductivos. Pasaron 15 años más hasta que en las Conferencias Internacionales realizadas en El Cairo y Beijing se ratificara el derecho a poder decidir de manera libre de coerción y violencia sobre la maternidad, entendida como un derecho de las mujeres.

En esta línea, el objetivo de este artículo es realizar un recorrido historiográfico por los debates respecto de los derechos sexuales y reproductivos desde mediados del siglo XX hasta la actualidad, en América Latina, haciendo hincapié en Argentina. Nos interesa señalar puntualmente dos momentos de inflexión: el momento en que la salud reproductiva pasó de la agenda de población a la agenda de los derechos humanos, y la propuesta conceptual de Brown (2014) respecto de los derechos sexuales y (no) reproductivos.

\section{Entre los debates poblacionales y los derechos humanos}

Durante la segunda mitad del siglo XX, las discusiones en torno a la salud sexual y reproductiva (SSR) se realizaron en el marco de los debates poblaciones que acontecían particularmente en Latinoamérica. En la década de 1950, esta cuestión ocupó un lugar importante en la agenda política internacional, ya que el crecimiento acelerado de la natalidad se vio acompañado por una caída de la mortalidad, lo que se reflejó rápidamente en un aumento marcado y sostenido de la población. En las discusiones sobre los cambios en la dinámica demográfica, con respecto a la forma de concebir el futuro desarrollo de las naciones (Pantelides, 1983, 1984), se podían distinguir dos posturas contrapuestas. Por un lado, se encontraban las opiniones de algunos intelectuales latinoamericanos que hacían foco en lo imperioso de poblar el continente y, por lo tanto, pensaban en la necesidad de aumentar la población, ya que, frente a una posible intervención militar, era fundamental que el país estuviera poblado (Felitti, 2007, 2008). En esta línea, los intelectuales y políticos latinoamericanos identificaron en la creación de instituciones y organismos internacionales preocupados por el desarrollo poblacional de los países pobres del Tercer Mundo (apoyadas por Estados Unidos), programas de promoción de la planificación familiar (PF) como forma de encubrir políticas imperialistas de control geopolítico en la región (Felitti, 2008: 518).

Por lo tanto, los gobiernos latinoamericanos cuestionaron el diagnóstico y la intervención estadounidense en la dinámica demográfica, ya que este análisis no tenía en cuenta que las variables de población y desarrollo estaban relacionadas con factores sociales, económicos y culturales que presentaban diferencias significativas en cada región, sobre todo entre América Latina, Asia y África. A su vez, estos intelectuales, apoyados en la teoría de la dependencia, vieron las recomendaciones estadounidenses sobre la necesidad de limitar los nacimientos para disminuir el crecimiento poblacional, como una nueva forma de imperialismo que no buscaba solucionar los problemas del desarrollo, sino que en realidad condicionaba la soberanía de los Estados subdesarrollados respondiendo a las necesidades geopolíticas de los países centrales (Felitti, 2008: 518).

Por otro lado, las opiniones a favor del control de la natalidad hacían hincapié en que, en medio de la crisis 
mundial propia de la post-guerra, crecía el temor neomalthusiano a la falta de recursos y la incapacidad para satisfacer necesidades sociales básicas como la educación y la alimentación, y la única solución posible era la regulación del crecimiento poblacional (Felitti, 2007, 2008).

Sin embargo, en ambas posturas, la opinión, la necesidad, el deseo o la salud de las mujeres, no era una variable a considerar. Lo que nos interesa preguntarnos aquí es ¿cómo fue el proceso socio-político que permitió incluir estos aspectos en el debate? Al constituir dos campos ajenos, en el contexto internacional no fue tarea sencilla lograr incorporar en las políticas públicas de población el tema de la salud de las mujeres como un derecho. Fue necesario un largo recorrido, hasta llegar a un consenso internacional sobre el derecho de las mujeres a poder decidir cuántos hijos tener y cuándo, libres de cualquier signo de coerción o violencia.

Históricamente, podemos rastrear que dicho proceso es fruto de la Declaración Universal de los Derechos Humanos firmada en 1948, donde se plasmó que el derecho a la libertad, la seguridad, la integridad física, la libertad de expresión, de pensamiento, de propiedad, entre otros, eran derechos irrenunciables de todas las personas. Con esta declaración como base jurídica, en 1968 la Conferencia Internacional de Derechos Humanos convocada por la Organización de las Naciones Unidas (ONU), resolvió que el número y el momento de las procreaciones son derechos de las personas y por lo tanto es necesario que toda la población, pero especialmente las mujeres, tengan un acceso igualitario a la información y cuenten con los servicios de salud disponibles para poder llevar adelante sus decisiones reproductivas. Posteriormente, en 1974, la Conferencia Internacional de Población realizada en Bucarest, ratificó que la procreación (o la no-procreación) es un derecho de todas las personas y parejas. A su vez, en 1979, la Convención para la Eliminación de todas las Formas de Discriminación hacia las Mujeres (CEDAW) reconoció, entre otras cosas, el derecho de las mujeres a contar con servicios de PF, y de educación, información y salud en la materia (De Barbieri, 2000).

Sin embargo, debemos aclarar que dichas afirmaciones se dieron en el plano declarativo y no se cristalizaron directamente en políticas públicas. Por ende, durante las décadas de 1970 y 1980, frente a la acumulación de abusos y violaciones de los derechos de las mujeres a la libre procreación y derechos de las parejas, los movimientos internacionales sobre la salud de las mujeres siguieron dos líneas (De Barbieri, 2000).

Una de ellas se dedicó a explorar los procesos de salud y enfermedad femeninos, la creación de servicios de SSR, y su implementación a partir del diseño de políticas públicas. Otra línea se dedicó a darle contenido jurídico a la expresión "derechos reproductivos", término que recién se consensuó en la década de 1980 a partir del I Encuentro Internacional de Salud de la Mujer realizado en Ámsterdam. A continuación, en 1987, un trabajo realizado por la Organización Mundial de la Salud, incorporó la noción de Salud Reproductiva como "un estado general de bienestar físico, mental y social, y no una mera ausencia de enfermedades o dolencias, en todos los aspectos relacionados al sistema reproductivo, sus funciones y procesos. Consecuentemente, la salud reproductiva implica la capacidad de disfrutar una vida sexual satisfactoria, sin riesgos para procrear, y con la libertad para poder escoger entre hacerlo o no, cuándo y con qué frecuencia” (Cepeda, 2008: 27).

Con estos antecedentes, en la década siguiente se explicitaron las definiciones de salud y derechos reproductivos. En la Conferencia Internacional de Población y Desarrollo de la ONU, realizada en El Cairo en 1994, el plan de acción afirmaba que

"Promover la equidad y la igualdad de los sexos y los derechos de la mujer, así como eliminar la violencia de todo tipo contra la mujer y asegurarse de que sea ella quien controle su propia fecundidad son la piedra angular de los programas de población y desarrollo. [...] Toda persona tiene derecho al disfrute del más alto nivel posible de salud física y mental. Los estados deberían adoptar todas las medidas apropiadas para asegurar, en condiciones de igualdad entre hombres y mujeres, el acceso universal a los servicios de atención médica, incluidos los relacionados con la salud reproductiva, que incluye la planificación familiar y la salud sexual”. 
Al año siguiente, durante la IV Conferencia Internacional de las Mujeres, realizada en Beijing, se ratificó que el derecho a poder decidir de manera libre de coerción y violencia sobre la maternidad, era un derecho de todas las mujeres.

No obstante, resulta necesario analizar cómo estas discusiones y definiciones a nivel internacional repercutieron en Argentina. Aunque la década de 1960 resultó un momento de quiebre a partir de la revolución sexual que generó la píldora anticonceptiva como forma de regulación de la natalidad de las mujeres de manera fácil y económica, podemos observar que los gobiernos latinoamericanos seguían pensando que la natalidad y la fecundidad eran aspectos de los que debían ocuparse las políticas de población (Felitti, 2012). Sólo los movimientos feministas vieron en los anticonceptivos orales un símbolo de liberación y se comprometieron en la difusión de los mismos, como una forma de controlar la natalidad de una manera relativamente fácil, accesible y eficaz, que no intervenía en el acto sexual y podía utilizarse más allá del acuerdo masculino.

Frente al avance de los movimientos feministas en la difusión y el uso de métodos anticonceptivos (MAC), basados en la premisa de que las mujeres pudieran decidir sobre su maternidad, durante la década del '60 las discusiones en torno a la planificación de políticas demográficas hacían hincapié en la necesidad de resolver la caída de la natalidad en Argentina (Bellucci, 2014). En el caso de nuestro país, la reducción temprana de la fecundidad fue un rasgo distintivo con respecto a otros países de América Latina, ya que desde 1930 hasta mediados de 1990 las mujeres argentinas tuvieron un promedio de 3 hijos, número menor que otras mujeres de América Latina que comenzaron a disminuir el número hijos a partir de 1970 (López, 2000; López y Mario, 2009) $)^{1}$.

En esta línea, el Plan Nacional de Desarrollo y Seguridad para 1971-1975, con el Decreto 558/1970 para proyectar un amplio crecimiento poblacional "instaba a aumentar la natalidad y proteger a la familia numerosa, disminuir la mortalidad infantil y atender a la inmigración con una política selectiva” (Felitti, 2008: 520).

Esta política pro natalista se profundizó en 1974, cuando el tercer gobierno peronista (fiel a sus objetivos poblacionistas y, por lo tanto, amparado en la idea de que el desarrollo del país se veía empañado por la falta de población) impulsó el Decreto 659/1974 para regular la comercialización de la píldora anticonceptiva a partir de que se exigiera para su compra recetas por triplicado y se cerraran consultorios de PF en todo el país (Cepeda, 2008; Bellucci, 2014). Además de estas dos cláusulas coercitivas, el Decreto también contemplaba la realización de campañas de educación sanitaria para poder garantizar el acceso universal a la salud por parte de las mujeres, pero éstas no se llevaron adelante. No obstante, no se aplicó sistemáticamente el requisito de la receta por triplicado al momento de la adquisición de MAC hormonales, lo que generó una gran desigualdad entre las mujeres, ya que aquellas que se encontraban en círculos feministas o de mayor poder adquisitivo, pudieron acceder a métodos de control de la natalidad, mientras que las mujeres de los sectores más humildes no pudieron acceder a los mismos. Lo que sí se aplicó de manera universal para toda la población fue el cierre de los consultorios de PF, impidiendo que las mujeres de los sectores más humildes pudieran acceder a información sobre control de la fecundidad (Cepeda, 2008).

Al respecto de estas políticas públicas es necesario señalar que, más allá de todas las convenciones internacionales en las que se incorporó la noción de Salud Reproductiva y se explicitaron los derechos de las mujeres sobre la decisión individual de regular la procreación, en Argentina las medidas restrictivas de comercialización de anticonceptivos hormonales se mantuvieron vigentes hasta 1986. El Decreto impulsado en 1974 se mantuvo como política de Estado durante la dictadura cívico-militar argentina (1976-1982). A pesar del regreso de la democracia en 1983 con la realización de elecciones libres en las que asumió Raúl Alfonsín como presidente, el proceso de democratización de la sociedad fue paulatino durante toda esa década. En el ámbito de los derechos sexuales y reproductivos, recién en 1986, mediante un nuevo decreto, se anularon las medidas restrictivas de la década anterior y se reconoció el derecho de las parejas a decidir cuántos hijos tener y cuándo. Aunque la anulación de estas políticas no garantizó el acceso a MAC a todas las mujeres, sino que sólo significó el acceso a mujeres de sectores sociales que tuvieran la información y dispusieran del dinero para adquirirlos en las farmacias (Cepeda, 2008; Bellucci, 2014). 
La década de 1990 fue el escenario de grandes debates a nivel internacional (como se mencionó anteriormente con las conferencias del El Cairo 1994 y Beijing 1995). Latinoamérica particularmente vivió una década de implementación de un modelo político, económico y social neoliberal que, a grandes rasgos, profundizó las desigualdades sociales en todas las esferas de la vida. En el caso puntual de los derechos sexuales y reproductivos, aunque en Argentina la venta de MAC era libre en términos legales, no todas las mujeres podían acceder económicamente a ellos. Por lo tanto, frente a los índices de desocupación y pobreza que atravesaba el país, muchas mujeres se vieron imposibilitadas de acceder a los mismos. En el 2003, con la asunción de Néstor Kirchner, comenzó un proceso de desarrollo de políticas públicas de inclusión social que permitieron revertir, de alguna manera, las desigualdades sociales que se habían profundizado durante la década anterior (Muñoz, 2010).

Para ello, era necesario que el Estado se hiciera cargo de la necesidad de generar una política de distribución y acceso a la información, en materia de SSR, que alcanzara a las mujeres de todos los sectores sociales. Por lo tanto, las políticas públicas estatales no podían estar sólo apuntadas a políticas socio-demográficas o de inserción en un proceso geo-político mundial, sino que debían comenzar a pensarse a partir del ámbito de los derechos humanos (Brown, 2014).

Como consecuencia de estos debates y de conceptualizar a la SSR como el derecho a tener relaciones sexuales gratificantes, sin coerción, violencia, enfermedades de transmisión sexual, ni embarazos no deseados o planificados, y que estos aparecen como fundamentales para mantener y mejorar la salud, practicar el cuidado propio y del otro/a; en el año 2002 se sancionó la Ley 25.673 de SSR donde se proponía desarrollar una política sostenida que garantice el acceso a la información y los MAC de manera universal, gratuita y segura.

La reglamentación de esta Ley en el año 2003, fue el comienzo de un proceso de democratización del acceso a los MAC en todo el sistema de Atención Primaria de la Salud Argentino (Arce, 2010; Testa, 2007), pero continua en la actualidad ya que la fragmentación del sistema de salud público no permitió que dicha política llegara a todo el territorio de manera homogénea.

\section{Derechos sexuales y (no) reproductivos.}

En el apartado anterior, hemos abordado el concepto de SSR con el objetivo de analizar cómo los temas en materia de sexualidad y reproducción pasaron de formar parte de la agenda de población a la agenda de salud, para poder comprender luego cómo se llegó a la sanción de la Ley Nacional de SSR. A continuación, nos centraremos en enmarcar este trabajo en un enfoque de Derechos Humanos (Brown, 2014). Hay amplios debates dentro del feminismo sobre si encuadrar o no los Derechos Sexuales y Reproductivos como parte de los Derechos Humanos (Brown, 2014; Petracci y Ramos, 2006; Petracci y Pecheny, 2010, 2009; Bellucci, 2014; López y Pantelides, 2007), por lo tanto, este apartado se propone realizar un recorrido teórico por el concepto de SSR, desde una perspectiva que haga hincapié en éstos.

Durante las décadas de 1960 y 1970, los grupos feministas en Argentina, dieron una gran disputa teórica y social para poder separar la sexualidad de la procreación (Bellucci, 2014). Su intención era discutir el papel central de la mujer en la sociedad a partir de la maternidad, cuestionando el argumento evolucionista de que la mujer "estaba preparada biológicamente para serlo” (Brown, 2014). Para las feministas, esta asociación mujer-madre se sostenía sobre un pilar fundamental que habría que derribar: el patriarcado como base del sistema capitalista moderno que generaba la subordinación de las mujeres.

El postulado de la construcción de la diferencia sexual jerarquizada se remonta a los orígenes de la civilización occidental, donde aparece una dicotomía entre hombre y mujeres, en la cual el hombre está asociado a la cultura y la mujer a la naturaleza en función de su sexualidad y fertilidad (Foucault 1977, Fausto-Sterling 2000, Rubin 1989). En este sentido, Luciano Fabbri (2013) afirma que

"La fetichización biológica es uno de los pilares de la ideología patriarcal, ya que es internalizada por los sujetos de forma tal que actúa sobre la autoconciencia, impidiendo su cuestionamiento. El 
carácter supuestamente neutral y objetivo de las ciencias naturales y su metodología aséptica de investigación funciona como punta de lanza de esta ideología” (Fabbri 2013: 78).

La intención de las teóricas feministas de los '60 y '70 fue justamente desenmascarar y denunciar el patriarcado como un sistema de opresión social, cultural y económica donde las diferencias sexuales que se asumían como biológicas y naturales sostenían la subordinación de las mujeres a la dominación masculina (Millet, 1975; Lerner, 1990). Siguiendo estos debates feministas, entendemos por patriarcado a la organización social jerarquizada de los sexos basada en la dominación masculina sobre las mujeres, construyendo así una red de poder que se asienta y perpetúa en la tradición de un discurso biológico cristalizado en la legitimación de una diferenciación social.

Simultáneamente, mientas las feministas discutían la vinculación entre el patriarcado y el capitalismo moderno, en la Conferencia Internacional de los Derechos Humanos de 1968 se incluyó dentro del ámbito de los Derechos Humanos a los Derechos Reproductivos. Sin embargo, no fue sencilla su universalización y aplicación dentro de estos derechos (ejemplo de ello fue el decreto 659/74 de control de la natalidad impulsado en Argentina para regular la comercialización de píldoras anticonceptivas). Recién en el Programa de Acción de El Cairo (1994) se puede observar un avance sobre la consideración de los derechos de las mujeres dentro del ámbito de los derechos humanos, a partir de considerar la salud como un derecho básico. Por lo tanto, las propuestas de acción del programa incluían aspectos como la reducción de los índices de mortalidad materna y el acceso universal a la salud reproductiva. Incluir la salud reproductiva en este programa resultó un quiebre fundamental ya que fue la primera vez que aparecía dentro del marco de estos derechos (Brown, 2014: 179). Sin embargo, cabe destacar que los avances en la materia, en el caso de Argentina, se hicieron simplemente en el plano declarativo ya que, en las políticas concretas, al año siguiente de la Conferencia de El Cairo se incluyó en la reforma constitucional de 1994 el Derecho a la vida de la persona por nacer, lo que imposibilitó cualquier avance dentro de los debates parlamentarios de un proyecto de legalización del aborto.

Siguiendo el planteo que realizábamos en la introducción respecto de los alcances y limitaciones que tiene todo concepto, que es un terreno de disputa y que, por lo tanto, incluye y excluye definiciones, es que nos parece fundamental retomar la idea de Brown (2014) sobre la utilización del concepto de derechos (no) reproductivos y sexuales. Para ella, los debates en los años '70 en torno al concepto "derechos reproductivos" como derechos humanos tienen dos puntos de apoyo fundamentales: la lucha feminista por el aborto legal en los países más desarrollados y las resistencias a las políticas coercitivas de control de la población implementadas en los países subdesarrollados. Ambos puntos en realidad no se apoyan en la idea de reproducción, sino todo lo contrario, en la idea de la no-reproducción. Por lo tanto, la conceptualización de los derechos reproductivos, sigue poniendo en primer plano a la reproducción, y dejando de lado la idea de la anticoncepción y el aborto. Es por esto, que la autora decide agregar el (no), entre paréntesis, para poder interpelar la imagen de la mujer como madre, y poder dar cuenta que lo que se llama comúnmente "derechos reproductivos", en realidad alude a las demandas feministas de tener derecho a una maternidad elegida y al derecho de poder elegir no ser madre. $\mathrm{Y}$ al mismo tiempo, le parece necesario agregar que los derechos no son sólo (no) reproductivos sino también sexuales, ya que es necesario incluir y visibilizar el ejercicio de otras sexualidades no heterosexuales y no reproductivas (Brown, 2014: 182-183).

El debate que recupera la autora con la incorporación del concepto de derechos sexuales y (no) reproductivos es fundamental ya que pone el acento en una contradicción del otro concepto con el que está discutiendo: el concepto de Derechos Reproductivos entendido como un derecho humano básico y universal que implicaba

"la posibilidad de ejercer la sexualidad y la maternidad en libertad, sin riesgos para la salud y sin ser objeto de discriminación cultural, social y laboral [...], de tomar decisiones reproductivas basadas en información veraz y accesible, y en recibir la protección social necesaria para llevar adelante estas decisiones; (y que) para ello debe asegurarse el acceso a: anticoncepción segura y eficaz, atención y protección del embarazo y la maternidad, aborto seguro y legal, tratamientos para la esterilidad, servicios de prevención y tratamiento de enfermedades de trasmisión sexual” (AAVV 1997: 149). 
Sin embargo, todas las políticas públicas devenidas de este concepto se enfocan en mujeres adultas, heterosexuales, preferentemente con pareja y en edad de procrear: prevención de ITS, control génito-mamario, atención antes, durante y después del embarazo. Por lo tanto, los varones, las personas no heterosexuales, los niños y las niñas y los y las adolescentes, los adultos mayores, tienen muchas más dificultades de ser considerados en el marco de estas políticas (Brown, 2014).

Pero entonces nos preguntamos, ¿por qué existe esta contradicción entre lo que pretende incluir dicho concepto y lo que excluye conceptualmente? Brown (2014) considera que la conquista de estos derechos se hizo a partir del concepto de salud reproductiva por dos motivos: por un lado, porque el movimiento feminista y de mujeres no pudo prevenir los aspectos que dejaría afuera, y por otro lado, porque algunos sectores no acuerdan con la demanda de los derechos sexuales ni con la reivindicación del derecho al aborto. Sin embargo, para la autora,

"lo importante aquí es que los conceptos así como las políticas, tienen una trayectoria y son consecuencia de determinado contexto. [...] Si bien la noción de salud reproductiva es altamente problemática por las razones anteriormente expuestas, sin embargo suponen un mínimo exigible al Estado en términos de derechos históricamente negados a un conjunto de seres humanos (las mujeres) también históricamente vulnerabilizados” (Brown, 2014: 189).

Es a partir de la lectura de Brown (2014), que nos interesa pensar cómo en Argentina, el regreso de la democracia en 1983 fue fundamental para poner de relieve que durante la dictadura cívico-militar se excluyó de todo debate público y político a los derechos sexuales y, al mismo tiempo, se reprimió a cualquiera de las voces que se propusiera reclamarlos. Con respecto a esto, Pecheny y Petracci (2006) afirman que la transición a la democracia favoreció

"la apertura y ampliación del universo político: nuevos temas son susceptibles de deliberación, gran parte de las relaciones sociales son cuestionadas y nuevos espacios se vuelven terreno de acciones políticas. En particular, la reivindicación de derechos sexuales politiza relaciones sociales consideradas privadas o naturales, poniendo en cuestión los límites instituidos entre lo privado y lo público, y entre lo natural y lo social” (Pecheny y Petracci, 2006: 44).

A su vez, durante toda la década de 1990, las diferentes convenciones internacionales pusieron en debate temas referidos tanto a la SSR como a los derechos de las mujeres. Con respecto a estas declaraciones internacionales, en la reforma constitucional de 1994 se les asignó mayor status, sobre todo a la Convención sobre la Eliminación de todas las Formas de Discriminación contra la Mujer (CEDAW). Sin embargo, los cambios más significativos en materia de SSR se observan a partir de la década siguiente.

\section{Conclusiones}

Retomar este debate historiográfico respecto de la noción de derechos sexuales y (no) reproductivos, nos permite pensar en la importancia de recuperar la génesis de cada concepto para comprender su interpretación en la actualidad.

En este trabajo, hemos visto cómo el concepto de salud sexual y reproductiva pasó de la agenda de población a la de salud y, luego, se comprendió dentro de los derechos humanos. Sin embargo, aunque dicho paso fue sumamente significativo e implicó grandes avances para los derechos de las mujeres, el mismo movimiento feminista que lo impulsó no pudo prever aquellos aspectos que dicho concepto dejó afuera. Por lo tanto, resulta fundamental recuperar el posicionamiento de Brown respecto del uso del concepto de derechos sexuales y (no) reproductivos para ser realmente inclusivos en su definición y conceptualización.

En este sentido, esta comprensión nos permite entender lo trascendente de la Ley de Salud Sexual y Reproductiva del año 2002, ya que cristalizó los debates que se sucedieron al respecto en el país, dejando en un primer plano el 
derecho de las mujeres a poder decidir libremente cuántos hijos tener y cuándo, garantizando el acceso universal a los métodos anticonceptivos. Sin embargo, la Ley no contempla de derecho a la interrupción voluntaria del embarazo. Más allá de la coyuntura política por la que el proyecto de Ley presentado al respecto aún no se trató en el Congreso de la Nación Argentina, recuperar las ideas de Brown respecto de cómo el concepto de derechos sexuales y reproductivos dejó por fuera (al menos conceptualmente) muchas de las demandar feministas respecto del aborto, es también una forma de comprender cómo se han llevado adelante dichos debates en las últimas décadas.

\section{Notas}

1 Esta diferencia se ha asociado al uso de MAC tradicionales y a la influencia sociocultural de la población inmigrante europea (Torrado, 1993, 2003; Pantelides 1986, 1988, 1989).

\section{Referencias bibliográficas}

Arce, H. (2010). El sistema de salud: de dónde viene y hacia dónde va. Qué pasó en el mundo, en el país y en los hospitales durante el siglo XX. Por qué estamos como estamos, Buenos Aires, Prometeo libros.

Bellucci, M. (2014). Historia de una desobediencia. Aborto y feminismo, Bs. As., Capital Intelectual.

Brown, J. L. (2014). Mujeres y ciudadanía en Argentina: debates teóricos y políticos sobre derechos (no) reproductivos y sexuales, Bs. As., Teseo.

Cepeda, A. (2008). "Historiando las políticas de sexualidad y los derechos en Argentina: entre los cuentos de la cigüeña y la prohibición de la pastilla (1974-2006)”, en Prácticas del oficio. Investigación y reflexión en Ciencias Sociales, $\mathrm{N}^{\circ}$ 2., UNGS-IDES, Bs. As.

De Barbieri, T. (2000). "Derechos reproductivos y sexuales. Encrucijada en tiempos distintos", en Revista Mexicana de Sociología, vol. 62, nún 1, Universidad Nacional Autónoma de México.

Fabbri, L. (2013). Apuntes sobre feminismos y construcción de poder popular, Rosario, Puño y letra.

Fausto-Sterling, A. (2000). Cuerpos sexuados: la política del género y la construcción de la sexualidad, Barcelona, Editorial Melusina.

Felitti, K. (2007). "El debate médico sobre anticoncepción y aborto en Buenos Aires en los años sesenta del siglo XX”, en Dynamis, vol. 27. Buenos Aires.

Felitti, K. (2008). "Natalidad, soberanía y desarrollo: las medidas restrictivas a la planificación familiar en el tercer gobierno peronista (Argentina, 1973-1976)”, en Estudos Feministas, Florianópolis. Maio-agosto.

Felitti, K. (2012). La revolución de la píldora. Sexualidad y política en los sesenta, Bs. As., Edhasa.

Foucault, M. (1977). Historia de la sexualidad, Bs. As., Siglo XXI Editores.

Lerner, G. (1990). La creación del patriarcado, Barcelona, Editorial Crítica.

López, E. y Pantelides, E. A. (comps.) (2007). Aportes a la investigación social en salud sexual y reproductiva, Bs. As., CEDES, CENEP, AEPA, UNFPA.

López, E. (2000). "Los dichos y los hechos: formación de la familia y anticoncepción en mujeres pobres del Conurbano de Buenos Aires”, en Pantelides E. A. y Bott, S. (comps.). Reproducción, Salud y Sexualidad en América Latina, Bs. As., Biblios y Organización Mundial de la Salud. 
López, E. y Mario (2009). “La fecundidad en la Argentina: 1996-2006: convergencias y divergencias”, en Población, Año 2, N 4, Bs. As., Dirección Nacional de Población, Secretaría del Interior.

Millet, K. (1975). Política Sexual, México, Ed. Aguilar.

Muñoz, M. A. (2010). Sísifo en Argentina: orden, conflicto y sujetos políticos, México, Editorial Plaza y Valdes.

Pantelides, E. A. (1983). La transición demográfica en Argentina: un modelo no ortodoxo, en Desarrollo Económico, vol 22, $\mathrm{N}^{\circ}$ 88, Bs. As., IDES.

Pantelides, E. A. (1984). “The decline of fertility in Argentina, 1869-1947” (Tesis Doctoral), The University of Texas ay Austin, EEUU.

Pantelides, E. A. (1986). "Notas respecto a la posible influencia de la inmigración europea sobre la fecundidad argentina”, en Estudios Migratorios Latinoamericanos, Disponible en http://cemla.com/publicaciones/

Pantelides, E. A. (1988). “La transición demográfica argentina: un modelo no ortodoxo”, en Cuadernos de la CENEP, Buenos Aires.

Pantelides, E. A. (1989). “La fecundidad argentina desde mediados del siglo XX”, en Cuadernos de la CENEP, Buenos Aires.

Petracci, M. y Ramos, S. (comps.) (2006). La política pública de salud y derechos sexuales y reproductivos en la Argentina: Aportes para comprender su historia, Bs. As., CEDES.

Petracci, M. y Pecheny, M. (2010). Panorama de derechos sexuales y reproductivos, Bs. As., IIGG Instituto de Investigaciones Gino Germani.

Petracci, M. y Pecheny, M. (2009). “Panorama de derechos sexuales y reproductivos”, en Argumentos, Revista de crítica social, $\mathrm{N}^{\circ} 11$, CLACSO.

Rubin, G. (1989). “Reflexionando sobre el sexo: notas para una teoría radical de la sexualidad”, en Vance, C. (comp.). Placer y peligro. Explorando la sexualidad femenina, Madrid, Editorial Revolución.

Testa, M. (2006). Pensar en salud, Bs. As., Lugar Editorial.

Torrado, S. (1993). Procreación en la Argentina: hechos e ideas, Bs. As., Centro de Estudios de la Mujer.

Torrado, S. (2003). Historia de la familia en la Argentina moderna, Bs. As., Ediciones de la Flor. 